\title{
Renal functional reserve
}

\author{
Praveen Ratanasrimetha MD, Miguel Quirch MD, Sorot Phisitkul MD
}

\begin{abstract}
Serum creatinine and glomerular filtration rate (GFR) are the current standard tests to measure kidney function. The baseline GFR does not reflect full function of the kidney since human kidneys do not always work at full capacity. Similarly, serum creatinine is not a sensitive measure for kidney function or injury. In healthy individuals the GFR physiologically increases in response to certain stresses or stimuli, such as protein loading.

Renal functional reserve (RFR) is defined as the difference between the maximal glomerular filtration rate (generally determined after oral or intravenous protein loading) and the baseline glomerular filtration rate. The absence of a normal RFR can help identify patients who are more susceptible to kidney injury. The RFR is also important in patients who develop acute kidney injury and chronic kidney disease. Even though the GFR might return to a baseline level, there may be some loss of RFR which can make the patient more susceptible to another episode of kidney injury.

Acute kidney injury and chronic kidney disease are considered interconnected syndromes; each is a risk factor for the other. There are no current recommendations regarding the performance of routine determinations of RFR. Physicians should focus on clinical history and physical examination in patients with a history of prior episodes of acute kidney injury, monitor renal function, and avoid nephrotoxic insults.
\end{abstract}

Keywords: glomerular filtration rate, renal functional reserve, creatinine, acute kidney injury

\section{INTRODUCTION}

The current standard tests of kidney function are the serum creatinine level and glomerular filtration rate (GFR). The serum creatinine is the product of the creatinine clearance and the daily creatinine production. Daily creatinine production depends upon muscle mass which varies with multiple factors, primarily age, sex, and race. The serum creatinine provides an estimate of how well the kidney is functioning, but in the absence of any calculation or estimate of the GFR, it cannot predict what will happen to the kidney during clinical insults, such as exposure to nephrotoxic substances,

Corresponding author: Praveen Ratanasrimetha Contact Information: Praveen.ratanasrimetha@ ttuhsc.edu

DOI: $10.12746 /$ swrccc.v6i25.480 autoimmune complex deposition, and infections. The serum creatinine concentration does not sensitively detect changes in kidney function. ${ }^{1-3}$ For a person who is at risk of kidney injury, current serum creatinine measurements, especially when near normal, cannot tell who will develop acute kidney injury. The baseline GFR might not reflect the full anatomical and functional features, including reserve, in the kidney. ${ }^{1,4-6}$

Normally, the human kidney does not work at full capacity. Each individual is born with a different number of nephrons based on genetic and environmental factors. ${ }^{1}$ Glomerular filtration rate can increase in response to stress or stimuli, such as a protein load or the physiologic changes that occur in pregnancy. Multiple studies have shown that an assortment of protein loads can increase GFR; some studies have reported increases up to $62-81 \% .^{1,7-9}$ The change in GFR with a protein load is similar to the change in cardiac output with exercise. 


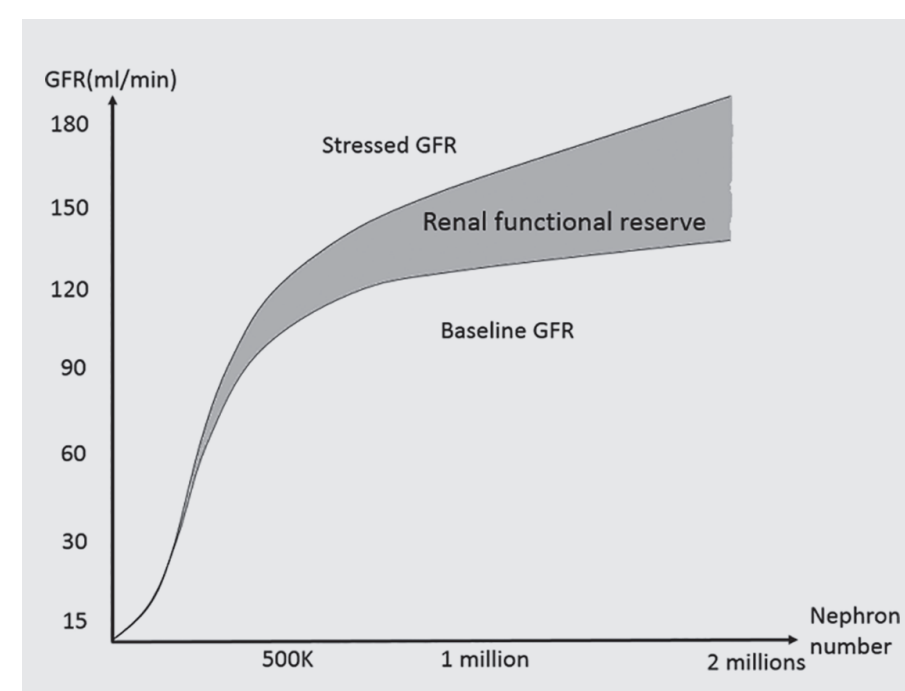

Figure 1. Relationship between baseline GFR and stressed GFR with nephron number. Renal functional reserve starts to decline with the loss of nephron number. ${ }^{10}$

Renal functional reserve (RFR) has been studied for several decades. It is defined as the difference between the baseline and maximum GFR, which is usually measured after oral or IV amino acid loading. ${ }^{1,8,9,11}$ Two people may have the same GFR, but one might be more susceptible to acute kidney injury $(\mathrm{AKI})$ than the other due to differences in renal reserve. The RFR acts as a sensitive method for the detection of subclinical kidney damage, and thus may help identify patients susceptible to kidney injury since it provides more information about whole kidney function and the remaining reserve..$^{4,6-8}$

In a patient who develops AKI, the baseline GFR may return to normal, but RFR may have been lost. This has long-term effects as the kidney is now more vulnerable to future episodes of $\mathrm{AKI} .{ }^{4}$ For this reason, some nephrologists consider a patient who has already recovered from AKI with a normal GFR but impaired RFR on stress testing as having chronic kidney disease (CKD). ${ }^{1,3,4,6,7,12}$

Renal stress testing is useful in predicting the risk of developing kidney injury since it uncovers loss of renal functional mass when there is no clinical evidence of kidney injury. ${ }^{7}$ The RFR has been used to predict risk of $\mathrm{AKI}$ in patient undergoing elective cardiac surgery, and it helped predict the development of AKI. ${ }^{13}$ This study showed that patients with reduced RFR preoperatively were more likely to develop AKI. Consequently, RFR can help physicians reduce risk of AKI by triggering the avoidance of nephrotoxic medications, hypotension, and the use of large volumes of contrast. ${ }^{13}$

\section{Physiology of Renal functional reserve}

Oral protein intake increases GFR. This effect occurs both in normal and impaired kidneys. An increase in GFR usually occurs in the first hour after protein load, and the maximum effect occurs around 2-2.5 hrs. ${ }^{1,2,5,8,9,11,12,14-18}$ Glomerular filtration rate can be increased by increase renal plasma flow (RPF) or filtration fraction (FF) as in this equation: GFR = $\mathrm{FF} \times \mathrm{RPF}$. The increased GFR after protein intake is a result of increased renal plasma flow without a change in filtration fraction. ${ }^{4}$ Three main mechanisms underlie this increased GFR. ${ }^{11}$

\section{Metabolic mechanisms}

Metabolic mechanisms are thought to involve amino acid stimulation of other metabolic processes in the kidney that include tubular sodium reabsorption and thus increased oxygen consumption by the kidney. $9,11,14,16$

\section{Humoral factors}

Numerous humoral factors might have important roles in RFR. Endothelium derived relaxing factors (such as nitric oxide), prostaglandins, and the renin-angiotensin-aldosterone system can alter renal hemodynamics. Nitric oxide causes renal vasodilation and an increase in GFR. In animal models, when nitric oxide was inhibited, there was no increase in RFR after amino acid infusion. ${ }^{16,17}$ The level of prostaglandins is not elevated after protein loading, but some studies have shown that indomethacin administration can blunt the effect of increased RFR after protein loading due to indomethacin's inhibitory effect on vasodilatory prostaglandins. $^{5,9}$ The renin-angiotensin-aldosterone system is another important system in regulating renal vasodilation or constriction. Angiotensin II has 
been studied extensively since it causes renal vasoconstriction and controls the renal vascular tone. .,11,17 $^{-17}$ Glucagon also increases the GFR, and multiple studies have demonstrated that elevated levels of glucagon and amino acids increase renal plasma flow. ${ }^{2,5,7}$

3. Intrinsic renal mechanisms (tubuloglomerular feedback, tubular transport mechanisms)

Intrinsic renal mechanisms are likely involved in tubuloglomerular feedback; however, these require intact function of both glomeruli and tubules. After a protein load, there is an increase in tubular amino acid absorption. Amino acids in the proximal tubule are co-transported with sodium so there is an increase in $\mathrm{NaCl}$ transport. This leads to a decrease in sodium delivery to the distal tubule and macula densa which provides feedback to release prostaglandin and nitric oxide. All of these effects cause renal vasodilatation. In patients with Fanconi syndrome (proximal tubule dysfunction) or with macula densa dysfunction, GFR does not increase after protein loads. ${ }^{9,11}$ This indicates that prostaglandins and nitric oxide have important roles in regulating GFR. ${ }^{9,11,16,17}$

Studies have shown that RFR decreases with age, but it occurs up to at least age 80 years. ${ }^{18}$ Renal functional reserve also decreases in advanced stage CKD. Renal functional reserve was shown to fall from $23.4 \%$ (normal population) to $6.7 \%$ in CKD stage four. ${ }^{3,18}$

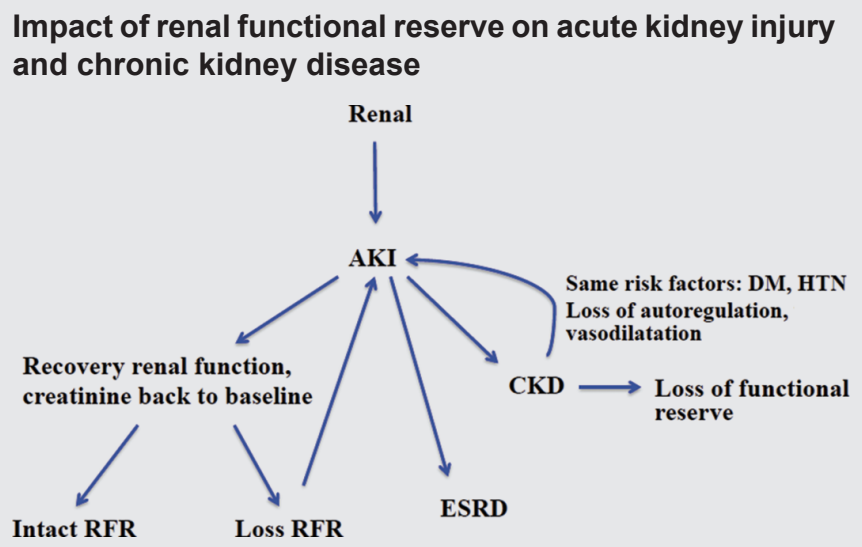

Figure 2. Relationship between renal functional reserve and development of AKI and CKD.
Donor nephrectomy is a good example of how RFR responds to an acute loss of renal function. A single kidney has a $20-40 \%$ increase in GFR within days after nephrectomy. Hyperfiltration and increased renal blood flow are responsible for this increase. ${ }^{19}$

Acute kidney injury is more complicated than nephrectomy. Acute kidney injury has four phases: 1) initiation, 2) extension, 3) maintenance, and 4) recovery. The initiation phase occurs when ischemia or toxin exposure results in cellular injury, particularly in renal tubular epithelial cells. The extension phase occurs when inflammatory factors, including a variety of chemokines and cytokines, are secreted. Renal vascular endothelial cell damage leads to vasoconstriction and prolongs the ischemia. ${ }^{20,21}$ In the maintenance phase, cells undergo repair, migration, apoptosis, proliferation, and restoration of renal blood flow. $^{22}$ In the recovery phase, cellular differentiation continues, the epithelial polarity is reestablished, and normal cellular and organ function return. . $^{23,24}$

In episodes of acute kidney injury, some patients have complete recovery while some progress to CKD. Physicians usually look at the baseline serum creatinine, but even when creatinine normalizes to baseline, patients may not recover to the same baseline RFR. In patients with less functional reserve, there is an increased risk of future AKI due to low nephron numbers, endothelial dysfunction, and abnormal vascular dilatation. ${ }^{1,4,10,24}$ In each episode of AKI, patients lose some nephrons. The remaining nephrons can develop adaptive hyperfiltration to maintain an intact GFR, but RFR will have been compromised. Maladaptive repair produces intrarenal hypertension, tubular hypertrophy and atrophy, tubulointerstitial fibrosis, progressive glomerular sclerosis and arteriosclerosis, and can lead to CKD. ${ }^{25}$

Chronic kidney disease patients may be at risk for the development of AKI. These patients often have other commonly accepted risk factors, e.g., DM, HTN, proteinuria, and old age in addition to CKD itself. The direct mechanisms of increased AKI risk in CKD patients include failure of autoregulation, abnormal vasodilatation, susceptibility to antihypertensive agents, and side effects of medication (such as diuretics). ${ }^{26}$ In kidney 
transplant patients, RFR can be used to identify susceptible donors and recipients for postoperative kidney injury. ${ }^{27}$

Overall RFR can be easily used in clinical practice. It could help identify patients who are susceptible to kidney injury and alert physicians to the potential for adverse kidney outcomes in patients with low RFRs. More studies of RFR are needed, and it could be easily incorporated into clinical trials.

\section{Conclusion}

Acute kidney injury and CKD are interconnected syndromes. Each is a risk factor for the other. The appearance of normal renal recovery from AKI may not reflect lost RFR which leads to increased susceptibility to future AKI. There is no current recommendation to do routine testing for RFR after an episode of AKI. We emphasize that physicians should get detailed histories and physical examinations for past AKI episodes, even in patients with normal renal function. Physicians should more closely monitor renal function and drug side effects in these patients.

Article citation: Ratanasrimetha P, Quirch M, Phisitkul S. Renal functional reserve. The Southwest Respiratory and Critical Care Chronicles 2018;6(25):26-30

From: Department of Internal Medicine, Texas Tech University Health Sciences Center, Lubbock, TX

Submitted: $11 / 17 / 2017$

Accepted: 6/17/2018

Conflicts of interest: None

This work is licensed under a Creative Commons Attribution-ShareAlike 4.0 International License

\section{REFERENCES}

1. Sharma A, Mucino MJ, Ronco C. Renal functional reserve and renal recovery after acute kidney injury. Nepron Clin Pract 2014;127(1-4):94-100.

2. Musso CG. Renal reserve test: its methodology and significance. Saudi J Kidney Dis Transpl 2011;22(5):990-3.

3. Barai S, Gambhir S, Prasad N, et al. Functional renal reserve capacity in different stages of chronic kidney disease. Nephrology (Carlton) 2010;15(3):350-3.
4. Ronco C, Rosner MH. Acute kidney injury and residual renal function. Crit Care 2012;16(4):144.

5. Koyner JL, Chawla LS. Use of stress tests in evaluating kidney disease. Curr Opin Nephrol Hypertens 2017;26(1):31-5.

6. Ronco C, Ferrari F, Ricci Z. Recovery after acute kidney injury: a new prognostic dimension of the syndrome. Am J Respir Crit Care Med 2017;195(6):711-4.

7. Sharma A, Zaragoza JJ, Villa G, et al. Optimizing a kidney stress test to evaluate renal functional reserve. Clin Nephrol 2016;86(7):18-26.

8. Bosch JP, Lauer A, Glabman S. Short-term protein loading in assessment of patients with renal disease. Am J Med 1984;77(5):873-9.

9. Friedlander G, Blanchet $F$, Amiel C. Renal functional reserve. Toxicol Lett 1989;46(1-3):227-35.

10. Tsuboi N, Kanzaki G, Koike K, et al. Clinicopathological assessment of the nephron number. Clin Kidney J 2014;7(2):107-14.

11. Woods LL. Mechanisms of renal hemodynamic regulation in response to protein feeding. Kidney Int 1993;44(4):659-75.

12. Gaipov A, Solak Y, Zhampeissov N, et al. Renal functional reserve and renal hemodynamics in hypertensive patients. Ren Fail 2016;38(9):1391-7.

13. Husain-Syed F, Ferrari F, Sharma A, et al. Preoperative renal functional reserve predicts risk of acute kidney injury after cardiac operation. Ann Thorac Surg. 2018 Apr;105(4):1094-101.

14. Memoli B, Libetta C, Sabbatini M, et al. Renal functional reserve: its significance in normal and salt depletion conditions. Kidney Int 1991;40(6):1134-40.

15. Raes A, Donckerwolcke R, Craen M, et al. Renal hemodynamic changes and renal functional reserve in children with type I diabetes mellitus. Pediatr Nephrol 2007;22(11):1903-9

16. De Nicola L, Keiser JA, Blantz RC, et al. Angiotensin II and renal functional reserve in rats with Goldblatt hypertension. Hypertension 1992;19(6 Pt 2):790-4.

17. De Nicola L, Blantz RC, Gabbai FB. Renal functional reserve in treated and untreated hypertensive rats. Kidney Int 1991;40(3):406-12.

18. Fliser $D$, Zeier $M$, Nowack $R$, et al. Renal functional reserve in healthy elderly subjects. J Am Soc Nephrol 1993;3(7):1371-7.

19. Mueller TF, Luyckx VA. The natural history of residual renal function in transplant donors. J Am Soc Nephrol 2012; 23(9):1462-6.

20. Molitoris BA, Sutton TA. Endothelial injury and dysfunction: role in the extension phase of acute renal failure. Kidney Int 2004;66(2):496-9.

21. Conger JD, Robinette JB, Hammond WS. Differences in vascular reactivity in models of ischemic acute renal failure. Kidney Int 1991;39(6):1087-97. 
22. Bonegio R, Lieberthal W. Role of apoptosis in the pathogenesis of acute renal failure. Curr Opin Nephrol Hypertens 2002;11(3):301-8.

23. Humphreys BD, Bonventre JV. Mesenchymal stem cells in acute kidney injury. Annu Rev Med 2008;59:311-25.

24. Cantley LG. Adult stem cells in the repair of the injured renal tubule. Nat Clin Pract Nephrol 2005;1(1):22-32.

25. Ferenbach DA, Bonventre JV. Mechanisms of maladaptive repair after AKI leading to accelerated kidney ageing and CKD. Nat Rev Nephrol 2015;11(5):264-76.
26. Chawla LS, Eggers PW, Star RA, et al. Acute kidney injury and chronic kidney disease as interconnected syndromes. $\mathrm{N}$ Engl J Med 2014;371(1):58-66.

27. Spinelli A, Sharma A,Villa G, et al. Rationale for the evaluation of renal functional reserve in living kidney donors and recipients: a pilot study. Nepron 2017;135(4): $268-76$ 